\title{
TRUNCATION AND DUALITY RESULTS FOR HOPF IMAGE ALGEBRAS
}

\author{
TEODOR BANICA
}

\begin{abstract}
Associated to an Hadamard matrix $H \in M_{N}(\mathbb{C})$ is the spectral measure $\mu \in \mathcal{P}[0, N]$ of the corresponding Hopf image algebra, $A=C(G)$ with $G \subset S_{N}^{+}$. We study here a certain family of discrete measures $\mu^{r} \in \mathcal{P}[0, N]$, coming from the idempotent state theory of $G$, which converge in Cesàro limit to $\mu$. Our main result is a duality formula of type $\int_{0}^{N}(x / N)^{p} d \mu^{r}(x)=\int_{0}^{N}(x / N)^{r} d \nu^{p}(x)$, where $\mu^{r}, \nu^{r}$ are the truncations of the spectral measures $\mu, \nu$ associated to $H, H^{t}$. We prove as well, using these truncations $\mu^{r}, \nu^{r}$, that for any deformed Fourier matrix $H=F_{M} \otimes_{Q} F_{N}$ we have $\mu=\nu$.
\end{abstract}

\section{INTRODUCTION}

A complex Hadamard matrix is a square matrix $H \in M_{N}(\mathbb{C})$ whose entries are on the unit circle, $\left|H_{i j}\right|=1$, and whose rows are pairwise orthogonal. The basic example of such a matrix is the Fourier one, $F_{N}=\left(w^{i j}\right)$ with $w=e^{2 \pi i / N}$ :

$$
F_{N}=\left(\begin{array}{ccccc}
1 & 1 & 1 & \ldots & 1 \\
1 & w & w^{2} & \ldots & w^{N-1} \\
\ldots & \ldots & \ldots & \ldots & \ldots \\
1 & w^{N-1} & w^{2(N-1)} & \ldots & w^{(N-1)^{2}}
\end{array}\right)
$$

In general, the theory of complex Hadamard matrices can be regarded as a "nonstandard" branch of discrete Fourier analysis. For a number of potential applications to quantum physics and quantum information theory questions, see [4], 8], [10].

Each Hadamard matrix $H \in M_{N}(\mathbb{C})$ is known to produce a subfactor $M \subset R$ of the Murray-von Neumann hyperfinite factor $R$, having index $[R: M]=N$. The associated planar algebra $P=\left(P_{k}\right)$ has a direct description in terms of $H$, worked out in [7], and a key problem is that of computing the corresponding Poincaré series, given by:

$$
f(z)=\sum_{k=0}^{\infty} \operatorname{dim}\left(P_{k}\right) z^{k}
$$

An alternative approach to this question is via quantum groups [11], [12]. The idea is that associated to $H \in M_{N}(\mathbb{C})$ is a quantum subgroup $G \subset S_{N}^{+}$of Wang's quantum

2000 Mathematics Subject Classification. 46L65 (46L37).

Key words and phrases. Quantum permutation, Hadamard matrix. 
permutation group [9], constructed by using to the Hopf image method, developed in [2]. More precisely, $G \subset S_{N}^{+}$appears via a factorization diagram, as follows:

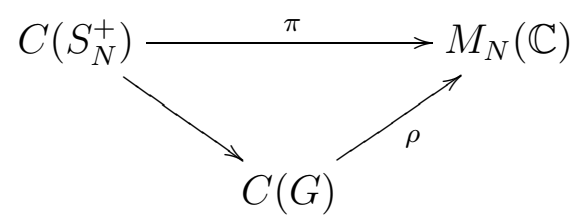

Here the upper arrow is defined by $\pi: u_{i j} \rightarrow P_{i j}=\operatorname{Proj}\left(H_{i} / H_{j}\right)$, where $u_{i j}$ are the standard generators of $C\left(S_{N}^{+}\right)$, and where $H_{1}, \ldots, H_{N} \in \mathbb{T}^{N}$ are the rows of $H$. The lower left arrow is by definition transpose to the embedding $G \subset S_{N}^{+}$, and the quantum group $G \subset S_{N}^{+}$itself is by definition the minimal one producing such a factorization.

With this notion in hand, the problem is that of computing the spectral measure $\mu$ of the main character $\chi: G \rightarrow \mathbb{C}$. This is indeed the same problem as above, because by Woronowicz's Tannakian duality [12], $f$ is the Stieltjes transform of $\mu$ :

$$
f(z)=\int_{G} \frac{1}{1-z \chi}
$$

Here, and in what follows, we use the integration theory developed in [11].

As a basic example, for a Fourier matrix $F_{N}$ the associated quantum group $G \subset S_{N}^{+}$is the cyclic group $\mathbb{Z}_{N}$, and we therefore have $\mu=\left(1-\frac{1}{N}\right) \delta_{0}+\frac{1}{N} \delta_{N}$ in this case. In general, however, the computation of $\mu$ is a quite difficult question. See [3].

In this paper we discuss a certain truncation procedure for the main spectral measure, coming from the idempotent state theory of the associated quantum group [3], [6]. Consider the following functionals, where $*$ is the convolution, $\psi * \phi=(\psi \otimes \phi) \Delta$ :

$$
\int_{G}^{r}=(t r \circ \rho)^{* r}
$$

The point with these functionals is that, as explained in [3], we have the following Cesàro limiting result, coming from the general results of Woronowicz in [11]:

$$
\int_{G} \varphi=\lim _{k \rightarrow \infty} \frac{1}{k} \sum_{r=1}^{k} \int_{G}^{r} \varphi
$$

This formula can be of course used in order to estimate or exactly compute the various integrals over $G$, and doing so will be the main idea in the present paper.

At the level of the main character, we have the following result:

Theorem A. The law $\chi$ with respect to $\int_{G}^{r}$ equals the law of the Gram matrix

$$
X_{i_{1} \ldots i_{r}, j_{1} \ldots j_{r}}=<\xi_{i_{1} \ldots i_{r}}, \xi_{j_{1} \ldots j_{r}}>
$$

of the norm one vectors $\xi_{i_{1} \ldots i_{r}}=\frac{1}{\sqrt{N}} \cdot \frac{H_{i_{1}}}{H_{i_{2}}} \otimes \ldots \otimes \frac{1}{\sqrt{N}} \cdot \frac{H_{i_{r}}}{H_{i_{1}}}$.

Here the law of $X$ is by definition its spectral measure, with respect to the trace. 
Observe that with $r \rightarrow \infty$, via the above-mentioned Cesàro limiting procedure, we obtain from the laws in Theorem A the spectral measure $\mu$ that we are interested in.

Our second, and main theoretical result, is as follows:

Theorem B. We have the moment/truncation duality formula

$$
\int_{G_{H}}^{r}\left(\frac{\chi}{N}\right)^{p}=\int_{G_{H^{t}}}^{p}\left(\frac{\chi}{N}\right)^{r}
$$

where $G_{H}, G_{H^{t}}$ are the quantum groups associated to $H, H^{t}$.

This formula, which is quite non-trivial, is probably quite interesting, in connection with the duality between the quantum groups $G_{H}, G_{\bar{H}}, G_{H^{t}}, G_{H^{*}}$ studied in [1].

As an illustration for the above methods, we will work out the case of the deformed Fourier matrices, $H=F_{N} \otimes_{Q} F_{M}$, with the following result:

Theorem C. For $H=F_{N} \otimes_{Q} F_{M}$ we have the self-duality formula

$$
\int_{G_{H}} \varphi(\chi)=\int_{G_{H^{t}}} \varphi(\chi)
$$

valid for any parameter matrix $Q \in M_{M \times N}(\mathbb{T})$.

The paper is organized as follows: 1-2 are preliminary sections, and in 3-4-5 we present the truncation procedure, and we prove Theorems A-B-C above.

Acknowledgements. I would like to thank Julien Bichon, Pierre Fima, Uwe Franz, Adam Skalski and Roland Vergnioux for several interesting discussions.

\section{HADAMARD MATRICES}

A complex Hadamard matrix is a matrix $H \in M_{N}(\mathbb{C})$ whose entries are on the unit circle, and whose rows are pairwise orthogonal. The basic example is the Fourier matrix, $F_{N}=\left(w^{i j}\right)$ with $w=e^{2 \pi i / N}$. More generally, we have as example the Fourier matrix $F_{G}=F_{N_{1}} \otimes \ldots \otimes F_{N_{k}}$ of any finite abelian group $G=\mathbb{Z}_{N_{1}} \times \ldots \times \mathbb{Z}_{N_{k}}$. See [8].

The complex Hadamard matrices are usually regarded modulo equivalence:

Definition 1.1. Two complex Hadamard matrices $H, K \in M_{N}(\mathbb{C})$ are called equivalent, and we write $H \sim K$, if one can pass from one to the other by permuting the rows and columns, or by multiplying these rows and columns by numbers in $\mathbb{T}$.

As explained in the introduction, each complex Hadamard matrix produces a subfactor $M \subset R$ of the Murray-von Neumann hyperfinite factor $R$, having index $[R: M]=N$, which can be understood in terms of quantum groups. Indeed, let us call "magic" any square matrix $u=\left(u_{i j}\right)$ whose entries are projections $\left(p=p^{2}=p^{*}\right)$, summing up to 1 on each row and column. We have then the following key definition, due to Wang [9]: 
Definition 1.2. $C\left(S_{N}^{+}\right)$is the universal $C^{*}$-algebra generated by the entries of a $N \times N$ magic matrix $u=\left(u_{i j}\right)$, with comultiplication, counit and antipode maps defined on the standard generators by $\Delta\left(u_{i j}\right)=\sum_{k} u_{i k} \otimes u_{k j}, \varepsilon\left(u_{i j}\right)=\delta_{i j}$ and $S\left(u_{i j}\right)=u_{j i}$.

As explained in [9], this algebra satisfies Woronowicz's axioms in [11, and so $S_{N}^{+}$is a compact quantum group, called quantum permutation group. Since the functions $v_{i j}$ : $S_{N} \rightarrow \mathbb{C}$ given by $v_{i j}(\sigma)=\delta_{i \sigma(j)}$ form a magic matrix, we have a quotient map $C\left(S_{N}^{+}\right) \rightarrow$ $C\left(S_{N}\right)$, which corresponds to an embedding $S_{N} \subset S_{N}^{+}$. This embedding is an isomorphism at $N=1,2,3$, but not at $N \geq 4$, where $S_{N}^{+}$is not finite. See [9].

The link with the Hadamard matrices comes from:

Definition 1.3. Associated to an Hadamard matrix $H \in M_{N}(\mathbb{T})$ is the minimal quantum group $G \subset S_{N}^{+}$producing a factorization of type

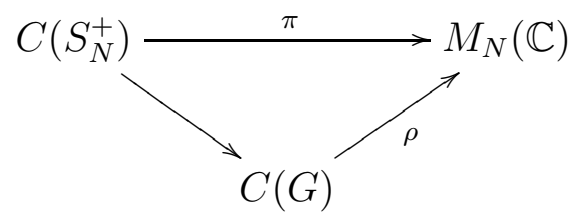

where $\pi: u_{i j} \rightarrow P_{i j}=\operatorname{Proj}\left(H_{i} / H_{j}\right)$, where $H_{1}, \ldots, H_{N} \in \mathbb{T}^{N}$ are the rows of $H$.

Here the fact that $\pi$ is indeed well-defined follows from the fact that $P=\left(P_{i j}\right)$ is magic, which comes from the fact that the rows of $H$ are pairwise orthogonal. As for the existence and uniqueness of the quantum group $G \subset S_{N}^{+}$as in the statement, this comes from Hopf algebra theory, by dividing $C\left(S_{N}^{+}\right)$by a suitable ideal. See [2].

At the level of examples, it is known that the Fourier matrix $F_{G}$ produces the group $G$ itself. In general, the computation of $G$ is a quite difficult question. See [3].

At a theoretical level, it is known that the above-mentioned subfactor $M \subset R$ associated to $H$ appears as a fixed point subfactor associated to $G$. See [1].

In what follows we will rather use a representation-theoretic formulation of this latter result. Let $u=\left(u_{i j}\right)$ be the fundamental representation of $G$.

Definition 1.4. We let $\mu \in \mathcal{P}[0, N]$ be the law of variable $\chi=\sum_{i} u_{i i}$, with respect to the Haar integration functional of $C(G)$.

Note that the main character $\chi=\sum_{i} u_{i i}$ being a sum of $N$ projections, we have the operator-theoretic formula $0 \leq \chi \leq N$, and so $\operatorname{supp}(\mu) \subset[0, N]$, as stated above.

Observe also that the moments of $\mu$ are integers, because we have the following computation, based on Woronowicz's general Peter-Weyl type results in [11]:

$$
\int_{0}^{N} x^{k} d \mu(x)=\int_{G} \operatorname{Tr}(u)^{k}=\int_{G} \operatorname{Tr}\left(u^{\otimes k}\right)=\operatorname{dim}\left(F i x\left(u^{\otimes k}\right)\right)
$$

The above moments, or rather the fixed point spaces appearing on the right, can be computed by using the following fundamental result, from [2]: 
Theorem 1.5. We have an equality of complex vector spaces

$$
F i x\left(u^{\otimes k}\right)=F i x\left(P^{\otimes k}\right)
$$

where for $X \in M_{N}(A)$ we set $X^{\otimes k}=\left(X_{i_{1} j_{1}} \ldots X_{i_{k} j_{k}}\right)_{i_{1} \ldots i_{k}, j_{1} \ldots j_{k}}$.

Now back to the subfactor problematics, it is known from [7] that the planar algebra associated to $H$ is given by $P_{k}=F i x\left(P^{\otimes k}\right)$. Thus, Theorem 1.5 tells us that the Poincaré series $f(z)=\sum_{k=0}^{\infty} \operatorname{dim}\left(P_{k}\right) z^{k}$ is nothing but the Stieltjes transform of $\mu$ :

$$
f(z)=\int_{G} \frac{1}{1-z \chi}
$$

Summarizing, modulo some standard correspondences, the main subfactor problem regarding $H$ consists in computing the spectral measure $\mu$ in Definition 1.4.

\section{Finiteness, DUALity}

We discuss in this section a key issue, namely the formulation of the duality between the quantum permutation groups associated to the matrices $H, \bar{H}, H^{t}, H^{*}$. Our claim is that the general scheme for this duality is, roughly speaking, as follows:

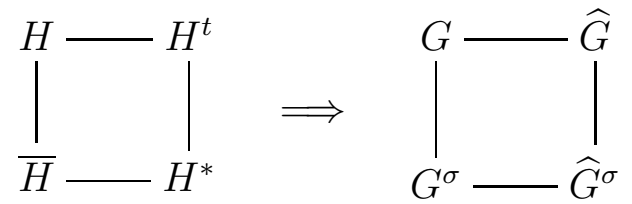

More precisely, this scheme fully works when the quantum groups are finite. In the general case the situation is more complicated, as explained in [1].

The results in [1, written some time ago, in the general context of vertex models, and without using the Hopf image formalism in [2], are in fact not very enlightening in the Hadamard matrix case. We will present below an updated approach. First, we have:

Proposition 2.1. The matrices $P=\left(P_{i j}\right)$ for $H, \bar{H}, H^{t}, H^{*}$ are related by:

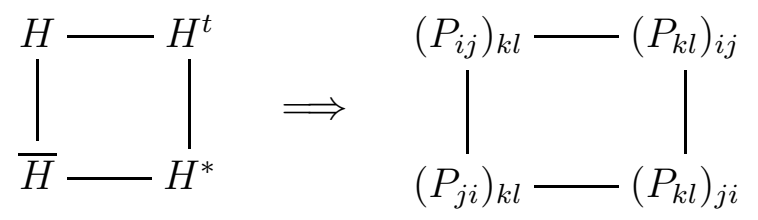

In addition, we have the formula $\left(P_{i j}\right)_{k l}=\left(P_{j i}\right)_{l k}$.

Proof. The magic matrix associated to $H$ is given by $P_{i j}=\operatorname{Proj}\left(H_{i} / H_{j}\right)$. Now since $H \rightarrow \bar{H}$ transforms $H_{i} / H_{j} \rightarrow H_{j} / H_{i}$, we conclude that the magic matrices $P^{H}, P^{\bar{H}}$ associated to $H, \bar{H}$ are related by the formula $P_{i j}^{H}=P_{j i}^{\bar{H}}$, as stated above.

In matrix notation, the formula for the matrix $P^{H}$ is as follows:

$$
\left(P_{i j}^{H}\right)_{k l}=\frac{1}{N} \cdot \frac{H_{i k} H_{j l}}{H_{i l} H_{j k}}
$$


Now by replacing $H \rightarrow H^{t}$, we obtain the following formula:

$$
\left(P_{i j}^{H^{t}}\right)_{k l}=\frac{1}{N} \cdot \frac{H_{k i} H_{l j}}{H_{l i} H_{k j}}=\left(P_{k l}^{H}\right)_{i j}
$$

Finally, the last assertion is clear from the above formula of $P^{H}$.

Let us compute now Hopf images. First, regarding the operation $H \rightarrow \bar{H}$, we have:

Proposition 2.2. The quantum groups associated to $H, \bar{H}$ are related by

$$
G_{\bar{H}}=G_{H}^{\sigma}
$$

where the Hopf algebra $C\left(G^{\sigma}\right)$ is $C(G)$ with comultiplication $\Sigma \Delta$, where $\Sigma$ is the flip.

Proof. Our claim is that, starting from a factorization for $H$ as in Definition 1.3 above, we can construct a factorization for $\bar{H}$, as follows:

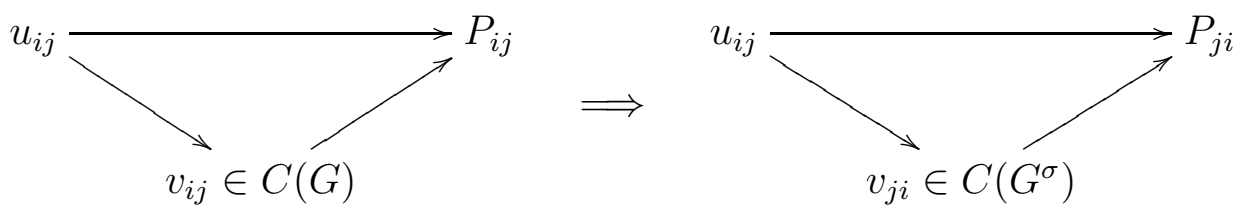

Indeed, observe first that since $v_{i j} \in C(G)$ are the coefficients of a corepresentation, then so are the elements $v_{j i} \in C\left(G^{\sigma}\right)$. Thus, in order to produce the factorization on the right, it is enough to take the diagram on the left, and compose at top left with the canonical map $C\left(S_{N}^{+}\right) \rightarrow C\left(S_{N}^{+\sigma}\right)$ given by $u_{i j} \rightarrow u_{j i}$, and we are done.

Let us investigate now the operation $H \rightarrow H^{t}$. We use the notion of dual of a finite quantum group, see e.g. [11]. The result here is as follows:

Theorem 2.3. The quantum groups associated to $H, H^{t}$ are related by usual duality,

$$
G_{H^{t}}=\widehat{G}_{H}
$$

provided that the quantum group $G_{H}$ is finite.

Proof. Our claim is that, starting from a factorization for $H$ as in Definition 1.3 above, we can construct a factorization for $H^{t}$, as follows:
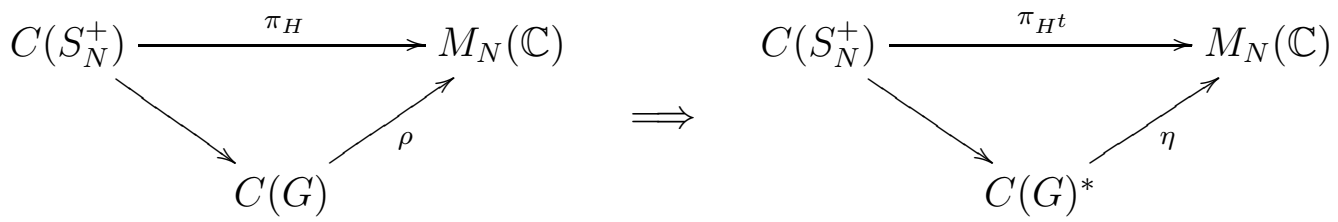

More precisely, having a factorization as the one on the left, let us set:

$$
\begin{aligned}
\eta(\varphi) & =\left(\varphi\left(v_{k l}\right)\right)_{k l} \\
w_{k l}(x) & =(\rho(x))_{k l}
\end{aligned}
$$


Our claim is that $\eta$ is a representation, $w$ is a corepresentation, and the factorization on the right holds indeed. Let us first check that $\eta$ is a representation:

$$
\begin{aligned}
\eta(\varphi \psi) & =\left(\phi \psi\left(v_{k l}\right)\right)_{k l}=\left((\varphi \otimes \psi) \Delta\left(v_{k l}\right)\right)_{k l}=\left(\sum_{a} \varphi\left(v_{k a}\right) \psi\left(v_{a l}\right)\right)_{k l}=\eta(\varphi) \eta(\psi) \\
\eta(\varepsilon) & =\left(\varepsilon\left(v_{k l}\right)\right)_{k l}=\left(\delta_{k l}\right)_{k l}=1 \\
\eta\left(\varphi^{*}\right) & =\left(\varphi^{*}\left(v_{k l}\right)\right)_{k l}=\left(\overline{\varphi\left(S\left(v_{k l}^{*}\right)\right)}\right)_{k l}=\left(\overline{\varphi\left(v_{l k}\right)}\right)_{k l}=\eta(\varphi)^{*}
\end{aligned}
$$

Let us check now the fact that $w$ is a corepresentation:

$$
\begin{aligned}
\left(\Delta w_{k l}\right)(x \otimes y) & =w_{k l}(x y)=\rho(x y)_{k l}=\sum_{i} \rho(x)_{k i} \rho(y)_{i l} \\
& =\sum_{i} w_{k i}(x) w_{i l}(y)=\left(\sum_{i} w_{k i} \otimes w_{i l}\right)(x \otimes y) \\
\varepsilon\left(w_{k l}\right) & =w_{k l}(1)=1_{k l}=\delta_{k l}
\end{aligned}
$$

We check now the fact that the above diagram commutes on the generators $u_{i j}$ :

$$
\eta\left(w_{a b}\right)=\left(w_{a b}\left(v_{k l}\right)\right)_{k l}=\left(\rho\left(v_{k l}\right)_{a b}\right)_{k l}=\left(\left(P_{k l}^{H}\right)_{a b}\right)_{k l}=\left(\left(P_{a b}^{H^{t}}\right)_{k l}\right)_{k l}=P_{a b}^{H^{t}}
$$

It remains to prove that $w$ is magic. We have the following formula:

$$
\begin{aligned}
w_{a_{0} a_{p}}\left(v_{i_{1} j_{1}} \ldots v_{i_{p} j_{p}}\right) & =\left(\Delta^{(p-1)} w_{a_{0} a_{p}}\right)\left(v_{i_{1} j_{1}} \otimes \ldots \otimes v_{i_{p} j_{p}}\right) \\
& =\sum_{a_{1} \ldots a_{p-1}} w_{a_{0} a_{1}}\left(v_{i_{1} j_{1}}\right) \ldots w_{a_{p-1} a_{p}}\left(v_{i_{p} j_{p}}\right) \\
& =\frac{1}{N^{p}} \sum_{a_{1} \ldots a_{p-1}} \frac{H_{i_{1} a_{0}} H_{j_{1} a_{1}}}{H_{i_{1} a_{1}} H_{j_{1} a_{0}}} \ldots \ldots \frac{H_{i_{p} a_{p-1}} H_{j_{p} a_{p}}}{H_{i_{p} a_{p}} H_{j_{p} a_{p-1}}}
\end{aligned}
$$

In order to check that each $w_{a b}$ is an idempotent, observe that we have:

$$
\begin{aligned}
w_{a_{0} a_{p}}^{2}\left(v_{i_{1} j_{1}} \ldots v_{i_{p} j_{p}}\right)= & \left(w_{a_{0} a_{p}} \otimes w_{a_{0} a_{p}}\right) \sum_{k_{1} \ldots k_{p}} v_{i_{1} k_{1}} \ldots v_{i_{p} k_{p}} \otimes v_{k_{1} j_{1}} \ldots v_{k_{p} j_{p}} \\
= & \frac{1}{N^{2 p}} \sum_{k_{1} \ldots k_{p}} \sum_{a_{1} \ldots a_{p-1}} \sum_{\alpha_{1} \ldots \alpha_{p-1}} \frac{H_{i_{1} a_{0}} H_{k_{1} a_{1}}}{H_{i_{1} a_{1}} H_{k_{1} a_{0}}} \ldots \frac{H_{i_{p} a_{p-1}} H_{k_{p} a_{p}}}{H_{i_{p} a_{p}} H_{k_{p} a_{p-1}}} \\
& \frac{H_{k_{1} a_{0}} H_{j_{1} \alpha_{1}}}{H_{k_{1} \alpha_{1}} H_{j_{1} a_{0}}} \ldots \frac{H_{k_{p} \alpha_{p-1}} H_{j_{p} a_{p}}}{H_{k_{p} a_{p}} H_{j_{p} \alpha_{p-1}}}
\end{aligned}
$$

The point now is that when summing over $k_{1}$ we obtain $N \delta_{a_{1} \alpha_{1}}$, then when summing over $k_{2}$ we obtain $N \delta_{a_{2} \alpha_{2}}$, and so on up to summing over $k_{p-1}$, where we obtain $N \delta_{a_{p-1} \alpha_{p-1}}$. 
Thus, after performing all these summations, what we are left with is:

$$
\begin{aligned}
w_{a_{0} a_{p}}^{2}\left(v_{i_{1} j_{1}} \ldots v_{i_{p} j_{p}}\right) & =\frac{1}{N^{p+1}} \sum_{k_{p}} \sum_{a_{1} \ldots a_{p-1}} \frac{H_{i_{1} a_{0}} H_{j_{1} a_{1}}}{H_{i_{1} a_{1}} H_{j_{1} a_{0}}} \ldots \ldots \frac{H_{i_{p} a_{p-1}} H_{k_{p} a_{p}}}{H_{i_{p} a_{p}} H_{k_{p} a_{p-1}}} \cdot \frac{H_{k_{p} a_{p-1}} H_{j_{p} a_{p}}}{H_{k_{p} a_{p}} H_{j_{p} a_{p-1}}} \\
& =\frac{1}{N^{p+1}} \sum_{k_{p}} \sum_{a_{1} \ldots a_{p-1}} \frac{H_{i_{1} a_{0}} H_{j_{1} a_{1}}}{H_{i_{1} a_{1}} H_{j_{1} a_{0}}} \ldots \ldots \frac{H_{i_{p} a_{p-1}} H_{j_{p} a_{p}}}{H_{i_{p} a_{p}} H_{j_{p} a_{p-1}}} \\
& =\frac{1}{N^{p}} \sum_{a_{1} \ldots a_{p-1}} \frac{H_{i_{1} a_{0}} H_{j_{1} a_{1}}}{H_{i_{1} a_{1}} H_{j_{1} a_{0}}} \ldots \frac{H_{i_{p} a_{p-1}} H_{j_{p} a_{p}}}{H_{i_{p} a_{p}} H_{j_{p} a_{p-1}}} \\
& =w_{a_{0} a_{p}}\left(v_{i_{1} j_{1}} \ldots v_{i_{p} j_{p}}\right)
\end{aligned}
$$

Regarding now the involutivity, the check here is simply:

$$
\begin{aligned}
w_{a_{0} a_{p}}^{*}\left(v_{i_{1} j_{1}} \ldots v_{i_{p} j_{p}}\right) & =\overline{w_{a_{0} a_{p}}\left(S\left(v_{i_{p} j_{p}} \ldots v_{i_{1} j_{1}}\right)\right)} \\
& =\overline{w_{a_{0} a_{p}}\left(v_{j_{1} i_{1}} \ldots v_{j_{p} i_{p}}\right)} \\
& =w_{a_{0} a_{p}}^{*}\left(v_{i_{1} j_{1}} \ldots v_{i_{p} j_{p}}\right)
\end{aligned}
$$

Finally, for checking the first "sum 1" condition, observe that we have:

$$
\sum_{a_{0}} w_{a_{0} a_{p}}\left(v_{i_{1} j_{1}} \ldots v_{i_{p} j_{p}}\right)=\frac{1}{N^{p}} \sum_{a_{0} \ldots a_{p-1}} \frac{H_{i_{1} a_{0}} H_{j_{1} a_{1}}}{H_{i_{1} a_{1}} H_{j_{1} a_{0}}} \ldots \ldots \frac{H_{i_{p} a_{p-1}} H_{j_{p} a_{p}}}{H_{i_{p} a_{p}} H_{j_{p} a_{p-1}}}
$$

The point now is that when summing over $a_{0}$ we obtain $N \delta_{i_{1} j_{1}}$, then when summing over $a_{1}$ we obtain $N \delta_{i_{2} j_{2}}$, and so on up to summing over $a_{p-1}$, where we obtain $N \delta_{i_{p} j_{p}}$. Thus, after performing all these summations, what we are left with is:

$$
\sum_{a_{0}} w_{a_{0} a_{p}}\left(v_{i_{1} j_{1}} \ldots v_{i_{p} j_{p}}\right)=\delta_{i_{1} j_{1}} \ldots \delta_{i_{p} j_{p}}=\varepsilon\left(v_{i_{1} j_{1}} \ldots v_{i_{p} j_{p}}\right)
$$

The proof of the other "sum 1" condition is similar, and this finishes the proof.

\section{The truncation procedure}

Let us go back now to the factorization in Definition 1.3. Regarding the Haar functional of the quantum group $G$, we have the following key result, from [3]:

Proposition 3.1. We have the Cesàro limiting formula

$$
\int_{G}=\lim _{k \rightarrow \infty} \frac{1}{k} \sum_{r=1}^{k} \int_{G}^{r}
$$

where the functionals at right are by definition given by $\int_{G}^{r}=(\operatorname{tr} \circ \rho)^{* r}$.

Regarding the functionals $\int_{G}^{r}$, their evaluation is a linear algebra problem. Several formulations of the problem were proposed in [3], and we will use here the following formula, which appears there, but in a somewhat technical form: 
Proposition 3.2. The functionals $\int_{G}^{r}=(\operatorname{tr} \circ \rho)^{* r}$ are given by

$$
\int_{G}^{r} u_{a_{1} b_{1}} \ldots u_{a_{p} b_{p}}=\left(T_{p}^{r}\right)_{a_{1} \ldots a_{p}, b_{1} \ldots b_{p}}
$$

where $\left(T_{p}\right)_{i_{1} \ldots i_{p}, j_{1} \ldots j_{p}}=\operatorname{tr}\left(P_{i_{1} j_{1}} \ldots P_{i_{p} j_{p}}\right)$, with $P_{i j}=\operatorname{Proj}\left(H_{i} / H_{j}\right)$.

Proof. With $a_{s}=i_{s}^{0}$ and $b_{s}=i_{s}^{r+1}$, we have the following computation:

$$
\begin{aligned}
\int_{G}^{r} u_{a_{1} b_{1}} \ldots u_{a_{p} b_{p}} & =(\operatorname{tr} \circ \rho)^{\otimes r} \Delta^{(r)}\left(u_{i_{1}^{0} i_{1}^{r+1}} \ldots u_{i_{p}^{0} i_{p}^{r+1}}\right) \\
& =(\operatorname{tr} \circ \rho)^{\otimes r} \sum_{i_{1}^{1} \ldots i_{p}^{r}} u_{i_{1}^{0} i_{1}^{1}} \ldots u_{i_{p}^{0} i_{p}^{1}} \otimes \ldots \ldots \otimes u_{i_{1}^{r} u_{1}^{r+1} \ldots u_{i_{p}^{r} i_{p}^{r+1}}} \\
& =\operatorname{tr} \otimes r \sum_{i_{1}^{1} \ldots i_{p}^{r}} P_{i_{1}^{0} i_{1}^{1}} \ldots P_{i_{p}^{0} i_{p}^{1}} \otimes \ldots . P_{i_{1}^{r} i_{1}^{r+1}} \ldots P_{i_{p}^{r} i_{p}^{r+1}}
\end{aligned}
$$

On the other hand, we have as well the following computation:

$$
\begin{aligned}
\left(T_{p}^{r}\right)_{a_{1} \ldots a_{p}, b_{1} \ldots b_{p}} & =\sum_{i_{1}^{1} \ldots i_{p}^{r}}\left(T_{p}\right)_{i_{1}^{0} \ldots i_{p}^{0}, i_{1}^{1} \ldots i_{p}^{1}} \ldots \ldots\left(T_{p}\right)_{i_{1}^{r} \ldots i_{p}^{r}, i_{1}^{r+1} \ldots i_{p}^{r+1}} \\
& =\sum_{i_{1}^{1} \ldots i_{p}^{r}} \operatorname{tr}\left(P_{i_{1}^{0} i_{1}^{1}} \ldots P_{i_{p}^{0} i_{p}}\right) \ldots \ldots \operatorname{tr}\left(P_{i_{1}^{r} i_{1}^{r+1}} \ldots P_{i_{p}^{r} i_{p}^{r+1}}\right) \\
& =\operatorname{tr}^{\otimes r} \sum_{i_{1}^{1} \ldots i_{p}^{r}} P_{i_{1}^{0} i_{1}^{1}} \ldots P_{i_{p}^{0} i_{p}^{1}} \otimes \ldots \otimes P_{i_{1}^{r} i_{1}^{r+1} \ldots P_{i_{p}^{r} i_{p}^{r+1}}}
\end{aligned}
$$

Thus we have obtained the formula in the statement, and we are done.

We can now define the truncations of $\mu$, as follows:

Proposition 3.3. Let $\mu^{r}$ be the law of $\chi$ with respect to $\int_{G}^{r}=(\operatorname{tr} \circ \rho)^{* r}$.

(1) $\mu^{r}$ is a probability measure on $[0, N]$.

(2) We have the formula $\mu=\lim _{k \rightarrow \infty} \frac{1}{k} \sum_{r=1}^{k} \mu^{r}$.

(3) The moments of $\mu^{r}$ are the numbers $c_{p}^{r}=\operatorname{Tr}\left(T_{p}^{r}\right)$.

Proof. (1) The fact that $\mu^{r}$ is indeed a probability measure follows from the fact that the linear form $(\operatorname{tr} \circ \rho)^{* r}: C(G) \rightarrow \mathbb{C}$ is a positive unital trace, and the assertion on the support comes from the fact that the main character $\chi$ is a sum of $N$ projections.

(2) This follows from Proposition 3.1, i.e. from the main result in 3 .

(3) This follows from Proposition 3.2 above, by summing over $a_{i}=b_{i}$.

Let us recall now that associated to a complex Hadamard matrix $H \in M_{N}(\mathbb{C})$ is its profile matrix, given by:

$$
Q_{a b, c d}=\frac{1}{N}\left\langle\frac{H_{a}}{H_{b}}, \frac{H_{c}}{H_{d}}\right\rangle=\frac{1}{N} \sum_{i} \frac{H_{i a} H_{i d}}{H_{i b} H_{i c}}
$$


With this notation, we have the following result:

Proposition 3.4. The measures $\mu^{r}$ have the following properties:

(1) $\mu^{0}=\delta_{N}$.

(2) $\mu^{1}=\left(1-\frac{1}{N}\right) \delta_{0}+\frac{1}{N} \delta_{N}$.

(3) $\mu^{2}=\operatorname{law}(S)$, where $S_{a b, c d}=\left|Q_{a b, c d}\right|^{2}$.

(4) For a Fourier matrix $F_{G}$ we have $\mu^{1}=\mu^{2}=\ldots=\mu$.

Proof. We use the formula $c_{p}^{r}=\operatorname{Tr}\left(T_{p}^{r}\right)$ from Proposition 3.3 (3) above.

(1) At $r=0$ we have $c_{p}^{0}=\operatorname{Tr}\left(T_{p}^{0}\right)=\operatorname{Tr}\left(I d_{N^{p}}\right)=N^{p}$, so $\mu^{0}=\delta_{N}$.

(2) At $r=1$, if we denote by $J$ the flat matrix $(1 / N)_{i j}$, we have indeed:

$$
c_{p}^{1}=\operatorname{Tr}\left(T_{p}\right)=\sum_{i_{1} \ldots i_{p}} \operatorname{tr}\left(P_{i_{1} i_{1}} \ldots P_{i_{p} i_{p}}\right)=\sum_{i_{1} \ldots i_{p}} \operatorname{tr}\left(J^{p}\right)=\sum_{i_{1} \ldots i_{p}} \operatorname{tr}(J)=N^{p-1}
$$

(3) This can be checked directly, and is also a consequence of Theorem 3.5 below.

(4) For a Fourier matrix the representation $\rho$ producing the factorization in Definition 1.3 is well-known to be faithful, and this gives the result.

In the general case now, we have the following result:

Theorem 3.5. We have $\mu^{r}=\operatorname{law}(X)$, where

$$
X_{a_{1} \ldots a_{r}, b_{1} \ldots b_{r}}=Q_{a_{1} b_{1}, a_{2} b_{2}} \ldots Q_{a_{r} b_{r}, a_{1} b_{1}}
$$

where $Q$ denotes as usual the profile matrix.

Proof. We compute the moments of $\mu^{r}$. We first have:

$$
\begin{aligned}
c_{p}^{r} & =\operatorname{Tr}\left(T_{p}^{r}\right)=\sum_{i^{1} \ldots i^{r}}\left(T_{p}\right)_{i^{1} i^{2}} \ldots\left(T_{p}\right)_{i^{r} i^{1}} \\
& =\sum_{i_{1}^{1} \ldots i_{p}^{r}}\left(T_{p}\right)_{i_{1}^{1} \ldots i_{p}^{1}, i_{1}^{2} \ldots i_{p}^{2}} \ldots \ldots\left(T_{p}\right)_{i_{1}^{r} \ldots i_{p}^{r}, i_{1}^{1} \ldots i_{p}^{1}} \\
& =\sum_{i_{1}^{1} \ldots i_{p}^{r}} \operatorname{tr}\left(P_{i_{1}^{1} i_{1}^{2}} \ldots P_{i_{p}^{1} i_{p}^{2}}\right) \ldots \operatorname{tr}\left(P_{i_{1}^{r} i_{1}^{1}} \ldots P_{i_{p}^{r} i_{p}^{1}}\right)
\end{aligned}
$$

In terms of $H$, we obtain the following formula:

$$
\begin{aligned}
c_{p}^{r} & =\frac{1}{N^{r}} \sum_{i_{1}^{1} \ldots i_{p}^{r}} \sum_{a_{1}^{1} \ldots a_{p}^{r}}\left(P_{i_{1}^{1} i_{1}^{2}}\right)_{a_{1}^{1} a_{2}^{1}} \ldots\left(P_{i_{p}^{1} i_{p}^{2}}\right)_{a_{p}^{1} a_{1}^{1}} \ldots \ldots\left(P_{i_{1}^{r} i_{1}^{1}}\right)_{a_{1}^{r} a_{2}^{r}} \ldots\left(P_{i_{p}^{r} i_{p}^{1}}\right)_{a_{p}^{r} a_{1}^{r}} \\
& =\frac{1}{N^{(p+1) r}} \sum_{i_{1}^{1} \ldots i_{p}^{r} a_{1}^{1} \ldots a_{p}^{r}} \frac{H_{i_{1}^{1} a_{1}^{1}} H_{i_{1}^{2} a_{2}^{1}}}{H_{i_{1}^{1} a_{2}^{1}} H_{i_{1}^{2} a_{1}^{1}}} \ldots \frac{H_{i_{p}^{1} a_{p}^{1}} H_{i_{p}^{2} a_{1}^{1}}}{H_{i_{p}^{1} a_{1}^{1}} H_{i_{p}^{2} a_{p}^{1}}} \ldots \frac{H_{i_{1}^{r} a_{1}^{r}} H_{i_{1}^{1} a_{2}^{r}}}{H_{i_{1}^{r} a_{2}^{r}} H_{i_{1}^{1} a_{1}^{r}}} \ldots \frac{H_{i_{p}^{r} a_{p}^{r}} H_{i_{p}^{1} a_{1}^{r}}}{H_{i_{p}^{r} a_{1}^{r}} H_{i_{p}^{1} a_{p}^{r}}}
\end{aligned}
$$


Now by changing the order of the summation, we obtain:

$$
\begin{aligned}
& c_{p}^{r}=\frac{1}{N^{(p+1) r}} \sum_{a_{1}^{1} \ldots a_{p}^{r}} \sum_{i_{1}^{1}} \frac{H_{i_{1}^{1} a_{1}^{1}} H_{i_{1}^{1} a_{2}^{r}}}{H_{i_{1}^{1} a_{2}^{1}} H_{i_{1}^{1} a_{1}^{r}}} \ldots \sum_{i_{1}^{r}} \frac{H_{i_{1}^{r} a_{2}^{r-1}} H_{i_{1}^{r} a_{1}^{r}}}{H_{i_{1}^{r} a_{1}^{r-1}} H_{i_{1}^{r} a_{2}^{r}}} \\
& \sum_{i_{p}^{1}} \frac{H_{i_{p}^{1} a_{p}^{1}} H_{i_{p}^{1} a_{1}^{r}}}{H_{i_{p}^{1} a_{1}^{1}} H_{i_{p}^{1} a_{p}^{r}}} \ldots \sum_{i_{p}^{r}} \frac{H_{i_{p}^{r} a_{1}^{r-1}} H_{i_{p}^{r} a_{p}^{r}}}{H_{i_{p}^{r} a_{p}^{r-1}} H_{i_{p}^{r} a_{1}^{r}}}
\end{aligned}
$$

In terms of $Q$, and then of the matrix $X$ in the statement, we get:

$$
\begin{aligned}
c_{p}^{r} & =\frac{1}{N^{r}} \sum_{a_{1}^{1} \ldots a_{p}^{r}}\left(Q_{a_{1}^{1} a_{2}^{1}, a_{1}^{r} a_{2}^{r}} \ldots Q_{a_{1}^{r} a_{2}^{r}, a_{1}^{r-1} a_{2}^{r-1}} \ldots \ldots\left(Q_{a_{p}^{1} a_{1}^{1}, a_{p}^{r} a_{1}^{r}} \ldots Q_{a_{p}^{r} a_{1}^{r}, a_{p}^{r-1} a_{1}^{r-1}}\right)\right. \\
& =\frac{1}{N^{r}} \sum_{a_{1}^{1} \ldots a_{p}^{r}} X_{a_{1}^{1} \ldots a_{1}^{r}, a_{2}^{1} \ldots a_{2}^{r}} \ldots \ldots X_{a_{p}^{1} \ldots a_{p}^{r}, a_{1}^{1} \ldots a_{1}^{r}} \\
& =\frac{1}{N^{r}} \operatorname{Tr}\left(X^{p}\right)=\operatorname{tr}\left(X^{p}\right)
\end{aligned}
$$

But this gives the formula in the statement, and we are done.

Observe that the above result covers the previous computations of $\mu^{0}, \mu^{1}, \mu^{2}$, and in particular the formula of $\mu^{2}$ in Proposition 3.4 (3). Indeed, at $r=2$ we have:

$$
X_{a b, c d}=Q_{a c, b d} Q_{b d, a c}=Q_{a b, c d} \overline{Q_{a b, c d}}=\left|Q_{a b, c d}\right|^{2}
$$

We will discuss in the next section some further interpretations of $\mu^{r}$.

\section{BASIC PROPERTIES, EXAMPLES}

Let us first take a closer look at the matrices $X$ appearing in Theorem 3.5. These are in fact Gram matrices, of certain norm one vectors:

Proposition 4.1. We have $\mu^{r}=\operatorname{law}(X)$, with $X_{a_{1} \ldots a_{r}, b_{1} \ldots b_{r}}=<\xi_{a_{1} \ldots a_{r}}, \xi_{b_{1} \ldots b_{r}}>$, where:

$$
\xi_{a_{1} \ldots a_{r}}=\frac{1}{\sqrt{N}} \cdot \frac{H_{a_{1}}}{H_{a_{2}}} \otimes \ldots \otimes \frac{1}{\sqrt{N}} \cdot \frac{H_{a_{r}}}{H_{a_{1}}}
$$

In addition, these vectors $\xi_{a_{1} \ldots a_{r}}$ are all of norm one.

Proof. The first assertion follows from the following computation:

$$
\begin{aligned}
X_{a_{1} \ldots a_{r}, b_{1} \ldots b_{r}} & =\frac{1}{N^{r}}\left\langle\frac{H_{a_{1}}}{H_{b_{1}}}, \frac{H_{a_{2}}}{H_{b_{2}}}\right\rangle \ldots\left\langle\frac{H_{a_{r}}}{H_{b_{r}}}, \frac{H_{a_{1}}}{H_{b_{1}}}\right\rangle \\
& =\frac{1}{N^{r}}\left\langle\frac{H_{a_{1}}}{H_{a_{2}}}, \frac{H_{b_{1}}}{H_{b_{2}}}\right\rangle \ldots\left\langle\frac{H_{a_{r}}}{H_{a_{1}}}, \frac{H_{b_{r}}}{H_{b_{1}}}\right\rangle \\
& =\frac{1}{N^{r}}\left\langle\frac{H_{a_{1}}}{H_{a_{2}}} \otimes \ldots \otimes \frac{H_{a_{r}}}{H_{a_{1}}}, \frac{H_{b_{1}}}{H_{b_{2}}} \otimes \ldots \otimes \frac{H_{b_{r}}}{H_{b_{1}}}\right\rangle
\end{aligned}
$$


As for the second assertion, this is clear from the formula of $\xi_{a_{1} \ldots a_{r}}$.

At the level of concrete examples now, we first have:

Proposition 4.2. For a Fourier matrix $H=F_{G}$ we have:

(1) $Q_{a b, c d}=\delta_{a+d, b+c}$.

(2) $X_{a_{1} \ldots a_{r}, b_{1} \ldots b_{r}}=\delta_{a_{1}-b_{1}, \ldots, a_{r}-b_{r}}$.

(3) $X^{2}=N X$, so $X / N$ is a projection.

Proof. We use the formulae $H_{i j} H_{i k}=H_{i, j+k}, \bar{H}_{i j}=H_{i,-j}$ and $\sum_{i} H_{i j}=N \delta_{j 0}$.

(1) We have indeed the following computation:

$$
Q_{a b, c d}=\frac{1}{N} \sum_{i} H_{i, a+d-b-c}=\delta_{a+d, b+c}
$$

(2) This follows from the following computation:

$$
\begin{aligned}
X_{a_{1} \ldots a_{r}, b_{1} \ldots b_{r}} & =\delta_{a_{1}+b_{2}, b_{1}+a_{2}} \ldots \delta_{a_{r}+b_{1}, b_{r}+a_{1}} \\
& =\delta_{a_{1}-b_{1}, a_{2}-b_{2}} \ldots \delta_{a_{r}-b-r, a_{1}-b_{1}} \\
& =\delta_{a_{1}-b_{1}, \ldots, a_{r}-b_{r}}
\end{aligned}
$$

(3) By using the formula in (2) above, we obtain:

$$
\begin{aligned}
\left(X^{2}\right)_{a_{1} \ldots a_{r}, b_{1} \ldots b_{r}} & =\sum_{c_{1} \ldots c_{r}} X_{a_{1} \ldots a_{r}, c_{1} \ldots c_{r}} X_{c_{1} \ldots c_{r}, b_{1} \ldots b_{r}} \\
& =\sum_{c_{1} \ldots c_{r}} \delta_{a_{1}-c_{1}, \ldots, a_{r}-c_{r}} \delta_{c_{1}-b_{1}, \ldots, c_{r}-b_{r}} \\
& =N \delta_{a_{1}-b_{1}, \ldots, a_{r}-b_{r}}=N X_{a_{1} \ldots a_{r}, b_{1} \ldots b_{r}}
\end{aligned}
$$

Thus $(X / N)^{2}=X / N$, and since $X / N$ is as well self-adjoint, it is a projection.

Another situation which is elementary is the tensor product one:

Proposition 4.3. Let $L=H \otimes K$.

(1) $Q_{i a j b, k c l d}^{L}=Q_{i j, k l}^{H} Q_{a b, c d}^{K}$.

(2) $X_{i_{1} a_{1} \ldots i_{r} a_{r}, j_{1} b_{1} \ldots j_{r} b_{r}}^{L}=X_{i_{1} \ldots i_{r}, j_{1} \ldots j_{r}}^{H} X_{a_{1} \ldots a_{r}, b_{1} \ldots b_{r}}^{K}$.

(3) $\mu_{L}^{r}=\mu_{H}^{r} * \mu_{K}^{r}$, for any $r \geq 0$.

Proof. (1) This follows from the following computation:

$$
\begin{aligned}
Q_{i a j b, k c l d}^{L} & =\frac{1}{N M} \sum_{m e} \frac{L_{m e, i a} L_{m e, l d}}{L_{m e, k c} L_{m e, j b}}=\frac{1}{N M} \sum_{m e} \frac{H_{m i} K_{e a} H_{m l} K_{l d}}{H_{m k} K_{e c} H_{m j} K_{e b}} \\
& =\frac{1}{N} \sum_{m} \frac{H_{m i} H_{m l}}{H_{m k} H_{m j}} \cdot \frac{1}{M} \sum_{e} \frac{K_{e a} K_{e d}}{K_{e c} K_{e b}}=Q_{i j, k l}^{H} Q_{a b, c d}^{K}
\end{aligned}
$$


(2) This follows from (2) above, because we have:

$$
\begin{aligned}
X_{i_{1} a_{1} \ldots i_{r} a_{r}, j_{1} b_{1} \ldots j_{r} b_{r}}^{L} & =Q_{i_{1} a_{1} j_{1} b_{1}, 1_{2} a_{2} j_{2} b_{2}}^{L} \ldots Q_{i_{r} a_{r} j_{r} b_{r}, i_{1} a_{1} j_{1} b_{1}}^{L} \\
& =Q_{i_{1} j_{1}, i_{2} j_{2}}^{H} Q_{a_{1} b_{1}, a_{2} b_{2}}^{K} \ldots Q_{i_{r} j_{r}, i_{1} j_{1}}^{H} Q_{a_{r} b_{r}, a_{1} b_{1}}^{K} \\
& =X_{i_{1} \ldots i_{r}, j_{1} \ldots j_{r}}^{H} X_{a_{1} \ldots a_{r}, b_{1} \ldots b_{r}}^{K}
\end{aligned}
$$

(3) This follows from (3) above, which tells us that, modulo certain standard indentifications, we have $X^{L}=X^{H} \otimes X^{K}$.

We will be back in section 5 below to the study of concrete examples. Now let us discuss some general duality issues. We have here:

Theorem 4.4. We have the moment/truncation duality formula

$$
\int_{G_{H}}^{r}\left(\frac{\chi}{N}\right)^{p}=\int_{G_{H^{t}}}^{p}\left(\frac{\chi}{N}\right)^{r}
$$

where $G_{H}, G_{H^{t}}$ are the quantum groups associated to $H, H^{t}$.

Proof. We use the following formula, from the proof of Theorem 3.5:

$$
c_{p}^{r}=\frac{1}{N^{(p+1) r}} \sum_{i_{1}^{1} \ldots i_{p}^{r}} \sum_{a_{1}^{1} \ldots a_{p}^{r}} \frac{H_{i_{1}^{1} a_{1}^{1}} H_{i_{1}^{2} a_{2}^{1}}}{H_{i_{1}^{1} a_{2}^{1}} H_{i_{1}^{2} a_{1}^{1}}} \ldots \frac{H_{i_{p}^{1} a_{p}^{1}} H_{i_{p}^{2} a_{1}^{1}}}{H_{i_{p}^{1} a_{1}^{1}} H_{i_{p}^{2} a_{p}^{1}}} \ldots \frac{H_{i_{1}^{r} a_{1}^{r}} H_{i_{1}^{1} a_{2}^{r}}}{H_{i_{1}^{r} a_{2}^{r}} H_{i_{1}^{1} a_{1}^{r}}} \ldots \frac{H_{i_{p}^{r} a_{p}^{r}} H_{i_{p}^{1} a_{1}^{r}}}{H_{i_{p}^{r} a_{1}^{r}} H_{i_{p}^{1} a_{p}^{r}}}
$$

By interchanging $p \leftrightarrow r$, and by transposing as well all the summation indices, according to the rules $i_{x}^{y} \rightarrow i_{y}^{x}$ and $a_{x}^{y} \rightarrow a_{y}^{x}$, we obtain the following formula:

$$
c_{r}^{p}=\frac{1}{N^{(r+1) p}} \sum_{i_{1}^{1} \ldots i_{p}^{r}} \sum_{a_{1}^{1} \ldots a_{p}^{r}} \frac{H_{i_{1}^{1} a_{1}^{1}} H_{i_{2}^{1} a_{1}^{2}}}{H_{i_{1}^{1} a_{1}^{2}} H_{i_{2}^{1} a_{1}^{1}}} \ldots \frac{H_{i_{1}^{r} a_{1}^{r}} H_{i_{2}^{r} a_{1}^{1}}}{H_{i_{1}^{r} a_{1}^{1}} H_{i_{2}^{r} a_{1}^{r}}} \ldots \frac{H_{i_{p}^{1} a_{p}^{1}} H_{i_{1}^{1} a_{p}^{2}}}{H_{i_{p}^{1} a_{p}^{2}} H_{i_{1}^{1} a_{p}^{1}}} \ldots \frac{H_{i_{p}^{r} a_{p}^{r}} H_{i_{1}^{r} a_{p}^{1}}}{H_{i_{p}^{r} a_{p}^{1}} H_{i_{1}^{r} a_{p}^{r}}}
$$

Now by interchaging all the summation indices, $i_{x}^{y} \leftrightarrow a_{x}^{y}$, we obtain:

$$
c_{r}^{p}=\frac{1}{N^{(r+1) p}} \sum_{i_{1}^{1} \ldots i_{p}^{r}} \sum_{a_{1}^{1} \ldots a_{p}^{r}} \frac{H_{a_{1}^{1} i_{1}^{r}} H_{a_{2}^{1} i_{1}^{2}}}{H_{a_{1}^{1} i_{1}^{2}} H_{a_{2}^{1} i_{1}^{1}}} \ldots \frac{H_{a_{1}^{r} i_{1}^{r}} H_{a_{2}^{r} i_{1}^{1}}}{H_{a_{1}^{r} i_{1}^{1}} H_{a_{2}^{r} i_{1}^{r}}} \ldots \frac{H_{a_{p}^{1} i_{p}^{1}} H_{a_{1}^{1} i_{p}^{2}}}{H_{a_{p}^{1} i_{p}^{2}} H_{a_{1}^{1} i_{p}^{1}}} \ldots \frac{H_{a_{p}^{r} i_{p}^{r}} H_{a_{1}^{r} i_{p}^{1}}}{H_{a_{p}^{r} i_{p}^{1}} H_{a_{1}^{r} i_{p}^{r}}}
$$

With $H \rightarrow H^{t}$, we obtain the following formula, this time for $H^{t}$ :

$$
c_{r}^{p}=\frac{1}{N^{(r+1) p}} \sum_{i_{1}^{1} \ldots i_{p}^{r}} \sum_{a_{1}^{1} \ldots a_{p}^{r}} \frac{H_{i_{1}^{1} a_{1}^{1}} H_{i_{1}^{2} a_{2}^{1}}}{H_{i_{1}^{2} a_{1}^{1}} H_{i_{1}^{1} a_{2}^{1}}} \ldots \frac{H_{i_{1}^{r} a_{1}^{r}} H_{i_{1}^{1} a_{2}^{r}}}{H_{i_{1}^{1} a_{1}^{r}} H_{i_{1}^{r} a_{2}^{r}}} \ldots \frac{H_{i_{p}^{1} a_{p}^{1}} H_{i_{p}^{2} a_{1}^{1}}}{H_{i_{p}^{2} a_{p}^{1}} H_{i_{p}^{1} a_{1}^{1}}} \ldots \frac{H_{i_{p}^{r} a_{p}^{r}} H_{i_{p}^{1} a_{1}^{r}}}{H_{i_{p}^{1} a_{p}^{r}} H_{i_{p}^{r} a_{1}^{r}}}
$$

The point now is that, modulo a permutation of terms, the quantity on the right is exactly the one as in the above formula of $c_{p}^{r}$. Thus, if we denote by $\alpha$ this quantity:

$$
c_{p}^{r}(H)=\frac{\alpha}{N^{(p+1) r}}, \quad c_{r}^{p}\left(H^{t}\right)=\frac{\alpha}{N^{(r+1) p}}
$$


Thus we have $N^{r} c_{p}^{r}(H)=N^{p} c_{r}^{p}\left(H^{t}\right)$, and by dividing by $N^{p+r}$, we obtain:

$$
\frac{c_{p}^{r}(H)}{N^{p}}=\frac{c_{r}^{p}\left(H^{t}\right)}{N^{r}}
$$

But this gives the formula in the statement, and we are done.

The above result shows that the normalized moments $\gamma_{p}^{r}=\frac{c_{p}^{r}}{N^{p}}$ are subject to the condition $\gamma_{p}^{r}(H)=\gamma_{r}^{p}\left(H^{t}\right)$. We have the following table of $\gamma_{p}^{r}$ numbers for $H$ :

$$
\left[\begin{array}{ccccc}
p \backslash r & 1 & 2 & r & \infty \\
1 & 1 / N & 1 / N & 1 / N & 1 / N \\
2 & 1 / N & \operatorname{tr}(S / N)^{2} & \operatorname{tr}(S / N)^{r} & c_{2} \\
p & 1 / N & \operatorname{tr}(S / N)^{p} & ? & c_{p} \\
\infty & 1 / N & c_{2} & \mu^{r}(1) & \mu(1)
\end{array}\right]
$$

Here we have used the well-known fact that for $\operatorname{supp}(\mu) \subset[0,1]$ we have $c_{p} \rightarrow \mu(1)$, something which is clear for discrete measures, and for continuous measures too.

Since the table for $H^{t}$ is transpose to the table of $H$, we obtain:

Proposition 4.5. $\mu_{H}(1)=\mu_{H^{t}}(1)$.

Proof. This follows indeed from Theorem 4.4, by letting $p, r \rightarrow \infty$.

Observe that this result recovers a bit of Theorem 2.3, because we have:

Proposition 4.6. For $G \subset S_{N}^{+}$finite we have $\mu(1)=\frac{1}{|G|}$.

Proof. The idea is to use the principal graph. So, let first $\Gamma$ be an arbitrary finite graph, with a distinguished vertex denoted 1 , let $A \in M_{M}(0,1)$ with $M=|\Gamma|$ be its adjacency matrix, set $N=\|\Gamma\|$, and let $\xi \in \mathbb{R}^{M}$ be a Perron-Frobenius eigenvector for $A$, known to be unique up to multiplication by a scalar. Our claim is that we have:

$$
\lim _{p \rightarrow \infty} \frac{\left(A^{p}\right)_{11}}{N^{p}}=\frac{\xi_{1}^{2}}{\|\xi\|^{2}}
$$

Indeed, if we choose an orthonormal basis of eigenvectors $\left(\xi^{i}\right)$, with $\xi^{1}=\xi /\|\xi\|$, and write $A=U D U^{t}$, with $U=\left[\xi^{1} \ldots \xi^{M}\right]$ and $D$ diagonal, then we have, as claimed:

$$
\left(A^{p}\right)_{11}=\left(U D^{p} U^{t}\right)_{11}=\sum_{k} U_{1 k}^{2} D_{k k}^{p} \simeq U_{11}^{2} N^{p}=\frac{\xi_{1}^{2}}{\|\xi\|^{2}} N^{p}
$$

Now back to our quantum group $G \subset S_{N}^{+}$, let $\Gamma$ be its principal graph, having as vertices the elements $r \in \operatorname{Irr}(G)$. The moments of $\mu$ being the numbers $c_{p}=\left(A^{p}\right)_{11}$, we have:

$$
\mu(1)=\lim _{p \rightarrow \infty} \frac{c_{p}}{N^{p}}=\lim _{p \rightarrow \infty} \frac{\left(A^{p}\right)_{11}}{N^{p}}=\frac{\xi_{1}^{2}}{\|\xi\|^{2}}
$$


On the other hand, it is known that with the normalization $\xi_{1}=1$, the entries of the Perron-Frobenius eigenvector are simply $\xi_{r}=\operatorname{dim}(r)$. Thus we have:

$$
\frac{\xi_{1}^{2}}{\|\xi\|^{2}}=\frac{1}{\sum_{r} \operatorname{dim}(r)^{2}}=\frac{1}{|G|}
$$

Together with the above formula of $\mu(1)$, this finishes the proof.

\section{Deformed Fourier MATRICES}

In this section we study the deformed Fourier matrices, $L=F_{M} \otimes_{Q} F_{N}$, constructed by Diţă in [5]. These matrices are defined by $L_{i a, j b}=Q_{i b}\left(F_{M}\right)_{i j}\left(F_{N}\right)_{a b}$.

We first have the following technical result:

Proposition 5.1. Let $H=F_{M} \otimes_{Q} F_{N}$, and set $R_{a b, c d}^{x}=\frac{1}{M} \sum_{m} w^{m x} \frac{Q_{m a} Q_{m d}}{Q_{m c} Q_{m b}}$.

(1) $Q_{i a j b, k c l d}=\delta_{a-b, c-d} R_{a b, c d}^{i+l-k-j}$.

(2) $X_{i_{1} a_{1} \ldots i_{r} a_{r}, j_{1} b_{1} \ldots j_{r} b_{r}}=\delta_{a_{1}-b_{1}, \ldots, a_{r}-b_{r}} R_{a_{1} b_{1}, a_{2} b_{2}}^{i_{1}+j_{2}-j_{1}-i_{2}} \ldots R_{a_{r} b_{r}, a_{1} b_{1}}^{i_{r}+j_{1}-j_{r}-i_{1}}$.

Proof. First, for a general deformation $H=K \otimes_{Q} L$, we have:

$$
\begin{aligned}
Q_{i a j b, k c l d} & =\frac{1}{M N} \sum_{m e} \frac{H_{m e, i a} H_{m e, l d}}{H_{m e, k c} H_{m e, j b}}=\frac{1}{M N} \sum_{m e} \frac{Q_{m a} K_{m i} L_{e a} Q_{m d} K_{m l} L_{l d}}{Q_{m c} K_{m k} L_{e c} Q_{m b} K_{m j} L_{e b}} \\
& =\frac{1}{M} \sum_{m} \frac{Q_{m a} Q_{m d}}{Q_{m c} Q_{m b}} \cdot \frac{K_{m i} K_{m l}}{K_{m k} K_{m j}} \cdot \frac{1}{N} \sum_{e} \frac{L_{e a} L_{e d}}{L_{e c} L_{e b}}
\end{aligned}
$$

Thus for a deformed Fourier matrix $H=F_{M} \otimes_{Q} F_{N}$ we have:

$$
Q_{i a j b, k c l d}=\delta_{a+d, b+c} \frac{1}{M} \sum_{m} \frac{Q_{m a} Q_{m d}}{Q_{m c} Q_{m b}} w^{m(i+l-k-j)}
$$

But this gives (1), and then (2), and we are done.

With the above formulae in hand, we can now state and prove:

Theorem 5.2. For the matrix $H=F_{M} \otimes_{Q} F_{N}$ we have

$$
\mu_{H}=\mu_{H^{t}}
$$

for any value of the parameter matrix $Q \in M_{M \times N}(\mathbb{T})$. 
Proof. We use the matrices $X, R$ constructed in Proposition 5.1 above. According to the result in Proposition 5.1 (2), we have the following formula:

$$
\begin{aligned}
c_{p}^{r}= & \frac{1}{N^{r}} \sum_{a_{1}^{1} \ldots a_{p}^{r}} X_{a_{1}^{1} \ldots a_{1}^{r}, a_{2}^{1} \ldots a_{2}^{r}} \ldots \ldots X_{a_{p}^{1} \ldots a_{p}^{r}, a_{1}^{1} \ldots a_{1}^{r}} \\
= & \frac{1}{N^{r}} \sum_{a_{1}^{1} \ldots a_{p}^{r}} \sum_{i_{1}^{1} \ldots i_{p}^{r}} \delta_{a_{1}^{1}-a_{2}^{1}, \ldots, a_{1}^{r}-a_{2}^{r}} R_{a_{1}^{1} a_{2}^{1}, a_{1}^{2} a_{2}^{2}}^{i_{1}^{1}+i_{2}^{2}-i_{2}^{2}-i_{1}^{1}} \ldots R_{a_{1}^{r} a_{2}^{r}, a_{1}^{1} a_{2}^{1}}^{i_{1}^{r}+i_{2}^{1}-i_{1}^{1}-r^{r}} \\
& \ldots \\
& \delta_{a_{p}^{1}-a_{1}^{1}, \ldots, a_{p}^{r}-a_{1}^{r}} R_{a_{p}^{1} a_{1}^{1}, a_{p}^{2} a_{1}^{2}}^{i_{1}^{1}+i_{1}^{2}-i_{1}^{1}-i_{p}^{2}} \ldots \ldots R_{a_{p}^{r} a_{1}^{r}, a_{p}^{1} a_{1}^{1}}^{i_{p}^{r}+i_{1}^{1}-i_{p}^{1}-i_{1}^{r}}
\end{aligned}
$$

Observe that the conditions on the $a$ indices, coming from the Kronecker symbols, state that the columns of $a=\left(a_{i}^{j}\right)$ must differ by vertical vectors of type $(s, \ldots, s)$.

Now let us compute the sum over $i$ indices, obtained by neglecting the Kronecker symbols. According to the formula of $R_{a b, c d}^{x}$ in Proposition 5.1, this is:

$$
\begin{aligned}
& S=\frac{1}{N^{p r}} \sum_{i_{1}^{1} \ldots i_{p}^{r}} \sum_{m_{1}^{1} \ldots m_{p}^{r}} w^{E(i, m)} \frac{Q_{m_{1}^{1} a_{1}^{1}} Q_{m_{1}^{1} a_{2}^{2}}}{Q_{m_{1}^{1} a_{2}^{1}} Q_{m_{1}^{1} a_{1}^{2}}} \ldots \ldots \frac{Q_{m_{1}^{r} a_{1}^{r}} Q_{m_{1}^{r} a_{2}^{1}}}{Q_{m_{1}^{r} a_{2}^{r}} Q_{m_{1}^{r} a_{1}^{1}}} \\
& \frac{Q_{m_{p}^{1} a_{p}^{1}} Q_{m_{p}^{1} a_{1}^{2}}}{Q_{m_{p}^{1} a_{1}^{1}} Q_{m_{p}^{1} a_{p}^{2}}} \ldots \cdots \frac{Q_{m_{p}^{r} a_{p}^{r}} Q_{m_{p}^{r} a_{1}^{1}}}{Q_{m_{p}^{r} a_{1}^{r}} Q_{m_{p}^{r} a_{p}^{1}}}
\end{aligned}
$$

Here the exponent appearing at right is given by:

$$
\begin{aligned}
E(i, m)= & m_{1}^{1}\left(i_{1}^{1}+i_{2}^{2}-i_{1}^{2}-i_{2}^{1}\right)+\ldots+m_{1}^{r}\left(i_{1}^{r}+i_{2}^{1}-i_{1}^{1}-i_{2}^{r}\right) \\
& \ldots \\
& +m_{p}^{1}\left(i_{p}^{1}+i_{1}^{2}-i_{p}^{2}-i_{1}^{1}\right)+\ldots+m_{p}^{r}\left(i_{p}^{r}+i_{1}^{1}-i_{p}^{1}-i_{1}^{r}\right)
\end{aligned}
$$

Now observe that this exponent can be written as:

$$
\begin{aligned}
E(i, m)= & i_{1}^{1}\left(m_{1}^{1}-m_{1}^{r}-m_{p}^{1}+m_{p}^{r}\right)+\ldots+i_{1}^{r}\left(m_{1}^{r}-m_{1}^{r-1}-m_{p}^{r}+m_{p}^{r-1}\right) \\
& \ldots \\
& +i_{p}^{1}\left(m_{p}^{1}-m_{p}^{r}-m_{p-1}^{1}+m_{p-1}^{r}\right)+\ldots+i_{p}^{r}\left(m_{p}^{r}-m_{p}^{r-1}-m_{p-1}^{r}+m_{p-1}^{r-1}\right)
\end{aligned}
$$

With this formula in hand, we can perform the sum over the $i$ indices, and the point if that the resulting condition on the $m$ indices will be exactly the same as the abovementioned condition on the $a$ indices. Thus, we obtain a formula as follows, where $\Delta($. 
is a certain product of Kronecker symbols:

$$
\begin{aligned}
c_{p}^{r}= & \frac{1}{N^{r}} \sum_{a_{1}^{1} \ldots a_{p}^{r}} \sum_{m_{1}^{1} \ldots m_{p}^{r}} \Delta(a) \Delta(m) \frac{Q_{m_{1}^{1} a_{1}^{1}} Q_{m_{1}^{1} a_{2}^{2}}}{Q_{m_{1}^{1} a_{2}^{1}} Q_{m_{1}^{1} a_{1}^{2}}} \ldots \ldots \frac{Q_{m_{1}^{r} a_{1}^{r}} Q_{m_{1}^{r} a_{2}^{1}}}{Q_{m_{1}^{r} a_{2}^{r}} Q_{m_{1}^{r} a_{1}^{1}}} \\
& \frac{Q_{m_{p}^{1} a_{p}^{1}} Q_{m_{p}^{1} a_{1}^{2}}}{Q_{m_{p}^{1} a_{1}^{1}} Q_{m_{p}^{1} a_{p}^{2}}} \ldots \frac{Q_{m_{p}^{r} a_{p}^{r}} Q_{m_{p}^{r} a_{1}^{1}}}{Q_{m_{p}^{r} a_{1}^{r}} Q_{m_{p}^{r} a_{p}^{1}}}
\end{aligned}
$$

The point now is that when replacing $H=F_{M} \otimes_{Q} F_{N}$ with its transpose matrix, $H^{t}=F_{N} \otimes_{Q^{t}} F_{M}$, we will obtain exactly the same formula, with $Q$ replaced by $Q^{t}$. But, with $a_{x}^{y} \leftrightarrow m_{x}^{y}$, this latter formula will be exactly the one above, and we are done.

\section{REFERENCES}

[1] T. Banica, Compact Kac algebras and commuting squares, J. Funct. Anal. 176 (2000), 80-99.

[2] T. Banica and J. Bichon, Hopf images and inner faithful representations, Glasg. Math. J. 52 (2010), 677-703.

[3] T. Banica, U. Franz and A. Skalski, Idempotent states and the inner linearity property, Bull. Pol. Acad. Sci. Math. 60 (2012), 123-132.

[4] I. Bengtsson, Three ways to look at mutually unbiased bases, AIP Conf. Proc. 889 (2007), 40-51.

[5] P. Diţă, Some results on the parametrization of complex Hadamard matrices, J. Phys. A 37 (2004), $5355-5374$.

[6] U. Franz and A. Skalski, On idempotent states on quantum groups, J. Algebra 322 (2009), 17741802.

[7] V.F.R. Jones and V.S. Sunder, Introduction to subfactors, Cambridge Univ. Press (1997).

[8] W. Tadej and K. Życzkowski, A concise guide to complex Hadamard matrices, Open Syst. Inf. Dyn. 13 (2006), 133-177.

[9] S. Wang, Quantum symmetry groups of finite spaces, Comm. Math. Phys. 195 (1998), 195-211.

[10] R.F. Werner, All teleportation and dense coding schemes, J. Phys. A 34 (2001), 7081-7094.

[11] S.L. Woronowicz, Compact matrix pseudogroups, Comm. Math. Phys. 111 (1987), 613-665.

[12] S.L. Woronowicz, Tannaka-Krein duality for compact matrix pseudogroups. Twisted SU(N) groups, Invent. Math. 93 (1988), 35-76.

T.B.: Department of Mathematics, Cergy-Pontoise University, 95000 Cergy-Pontoise, FRANCE. teodor.banica@u-cergy.fr 